\title{
Quantum Theory at Planck Scale, Dynamical Cosmological Term and Deformed Gravity
}

\author{
A. E. Shalyt-Margolin
}

National Centre of Particles and High Energy Physics, Bogdanovich Str. 153, Minsk, 220040, Belarus

\begin{abstract}
The dark energy problem is analyzed from the viewpoint of the time-varying cosmological term. It is demonstrated that, at least within the scope of the holographic principle, an experimental value of the vacuum energy density may be derived and may be tested quite well with a new small parameter arising in a quantum theory with the fundamental length on the order of Planck's length. Besides, within a theory proclaiming the existence of such fundamental length a high-energy deformation of the General Relativity for the space with horizon has been constructed. The obtained results have been applied to solving of the dark energy problem, making it possible to frame the following hypothesis: a dynamic cosmological term is a measure of deviation from a thermodynamic identity (the first law of thermodynamics) of the high-energy (Planck's) deformation of Einstein equations for horizon spaces in their thermodynamic interpretation.
\end{abstract}

Keywords Dark Energy, Fundamental Length, Deformation Parameter

\section{Introduction}

The cosmological constant $\Lambda$ is presently the most probable candidate for a role of dark energy. However, more and more researchers arrive at the idea that $\Lambda$ is a dynamic quantity $\Lambda=\Lambda(t)[1-3]$ rather than a constant. The idea is also supported by the inflation models pointing to the fact that in the early Universe the quantity was enormous[4], whereas this is evidently not so for the present-day Universe. If this is really the case, we have to appreciate time variations of this quantity and to realize why its current value conforms to the observable.

It should be recalled that in the initial calculations of $\Lambda$ with the use of a quantum field theory (QFT) it was cut-off at large (Planck) momenta[5,6] and, actually, implicitly it was QFT with the fundamental length on the order of Planck's $l_{p}$.

On the other hand, in the last decade numerous works devoted to a Quantum Field Theory (QFT) at Planck's scale[7-9] have been published(of course, the author has no pretensions of being exhaustive in his references). This interest stems from the facts that (i) at these scales it is expected to reveal the effects of a Quantum Gravity (QG), and this still unresolved theory is intriguing all the researchers engaged in the field of theoretical physics; (ii) modern accelerators, in particular LHC, have the capacity of achieving the energies at which some QG effects may be exhibited.

* Corresponding author:

a.shalyt@mail.ru (A. E. Shalyt-Margolin)

Published online at http://journal.sapub.org/ijtmp

Copyright (C) 2011 Scientific \& Academic Publishing. All Rights Reserved
Planck's scales undergoes changes associated with the appearance of additional parameters related to a minimal length (on the order of the Planck's length). As this takes place, the corresponding parameters are naturally considered as deformation parameters, i.e. the related quantum theories are considered as a high-energy deformation (at Planck's scales) of the well-known quantum field theory. The deformation is understood as an extension of a particular theory by inclusion of one or several additional parameters in such a way that the initial theory appears in the limiting transition[10].

The present work is a natural continuation of the previous papers of the author. The idea that a quantum theory at the Planck scales must involve the fundamental length has been put forward in the works devoted to a string theory fairly a long time ago[11]. But since it is still considered to be a tentative theory, some other indications have been required. Fortunately, by the present time numerous publications have suggested the appearance of the fundamental length in the Early Universe with the use of various approaches[12-15]. Of particular importance is the work[12], where on the basis of a simple gedanken experiment it is demonstrated that, with regard to the gravitational interactions (Planck's scales) exhibited in the Early Universe only, the Heisenberg Uncertainty Principle should be extended to the Generalized Uncertainty Principle[11-15]that in turn is bound to bring forth the fundamental length on the order of Planck's length. The advent of novel theories in physics of the Early Universe is associated with the introduction of new parameters, i.e. with a deformation of the well-known theories. Of course, in this case Heisenberg Algebra is subjected to the corresponding deformation too. Such a deformation may be based 
on the Generalized Uncertainty Principle (GUP)[16-18] as well as on the density matrix deformation[19-29].

At the same time, the above-mentioned new deformation parameters so far have not appeared in gravity despite the idea that they should. The situation is that no evident efforts have been undertaken to develop the high-energy (Planck's scale) gravity deformations including the deformation parameters introduced in a Quantum Theory of the Early Universe.

In this paper, with GUP held true, the possibility for the high-energy gravity deformation is considered for a specific case of Einstein's equations. As this takes place, the parameter $\alpha$ appearing in the Quantum Field Theory (QFT) with the UV cut off (fundamental length) produced by the density matrix deformation is used. There is no discrepancy of any kind as the deformation parameter in the GUP- produced Heisenberg algebra deformation is quite naturally expressed in terms of $\alpha$, and this will be shown later (Section 3). Besides, by its nature, $\alpha$ is better applicable to study the high-energy deformation of General Relativity because it is small, dimensionless (making series expansion more natural), and the corresponding representation of the Einstein's equations in its terms or its deformation appear simple. Structurally, the paper is as follows. Section 2 presents a short discussion o the notion of a dynamic cosmological term and also the general problem statement associated with its connection to the dark energy problem. In Section 3 the approaches to the deformation of a quantum theory at the Planck scales are briefly reviewed. In Section 4 the possibilities for derivation of a modern value of the dark energy in the assumption of the existing dynamic cosmological term $\Lambda(t)$ are analyzed using the standard approach to the cosmological constant $\Lambda[5,6]$. It is shown that only the validity of the holographic principle gives the required result. This Section is mainly based on the author's results[30,31]. In Section 5 it is shown how to interpret quantum corrections for the thermodynamic characteristics of black holes considering the deformation of a theory with the fundamental length[31]. Essentially new results are presented in Sections 5 and 6. A thermodynamic description of the General Relativity is used. The possibility for the high energy deformation of Einstein's equations is discussed within the scope of both equilibrium thermodynamics and non-equilibrium thermodynamics. In the latter case the approach is contemplated only in terms of a nature of the cosmological term. Moreover, in this case a more precise definition for the dependence of this term on the deformation parameter is possible. In Section 7 the derived results are applied to solve the dark energy problem. Based on the results obtained, in Conclusion the following hypothesis is framed: a dynamic cosmological term is a measure of deviation from the thermodynamic identity (the first law of thermodynamics) of the high-energy (Planck's) deformation of Einstein equations for horizon spaces in their thermodynamic interpretation.

\section{Dark Energy and Dynamical Cosmological Term}

The dark matter problem[32], along with the dark energy problem[33], is presently the basic problem in modern fundamental physics, astrophysics, and cosmology. Whereas for a nature of the first real hypotheses have been accepted already[34], the dark energy still remains enigmatic[35-38]. But it is the opinion of most researchers that dark energy represents the energy of the cosmic vacuum, its density being associated with the cosmological term $\Lambda$ in Einstein's equation[5,6,39]. In this respect an important reservation must be made - the point is that most common is the term cosmological constant. Actually, due to the Bianchi identities[40]

$$
\nabla_{\mu} G^{\mu}=0
$$

where $G^{\mu}{ }_{v}-$ Einstein equations

$$
G^{\mu}{ }_{v} \equiv R^{\mu}{ }_{v}-\frac{1}{2} \delta^{\mu}{ }_{v} R=\frac{8 \pi G}{c^{4}}\left[T^{\mu}{ }_{v}+\frac{c^{4} \Lambda}{8 \pi G} \delta^{\mu}{ }_{v}\right]
$$

the energy-momentum tensor $T^{\mu}{ }_{v}$ (energy-momentum density tensor) remains covariantly valid

$$
\nabla_{\mu} T^{\mu}{ }_{v}=0
$$

From whence it directly follows that the cosmological term $\Lambda$ is a constant.

But, as has been rightly noted in several publications (e.g.,[1-3]), conservation laws (3) are only regulating the energy-momentum exchange between the field sources and gravitational field and are liable to be violated if an independent energy source is existent in the Universe. Such a source may be associated with a time-varying cosmological term. So, in this case it is reasonable to consider $\Lambda=\Lambda(t)$.

Then the Bianchi identity (1) is replaced by the "generalized Bianchi identity"[2]

$$
\nabla_{\mu}\left[T_{v}^{\mu}+\Lambda_{v}^{\mu}\right]=0
$$

where $\Lambda^{\mu}{ }_{v}=\varepsilon_{\Lambda} \delta^{\mu}{ }_{v}$ is some energy-momentum tensor (referred to as the dark energy-momentum tensor [2]) related to the cosmological term, where

$$
\varepsilon_{\Lambda}=\frac{c^{4} \Lambda}{8 \pi G}
$$

is the corresponding energy density.

We are not interested in a diversity of the cosmological models involving the dynamic cosmological term $\Lambda=\Lambda(t)$ and different relationships between $\Lambda$ and $t$. Rather detailed references to such models have been made in the thesiswork[41]. Of interest is the problem: how can we obtain the observable value of $\Lambda=\Lambda_{\text {observ }}$ knowing that $\Lambda$ is dependent on $t$.

\section{Quantum Theory at Planck's Scale and Universal Deformation Parameter}

In the last twenty years the researchers have come to the understanding that studies of the Early Universe physics (extremely high - Planck's energies) necessitate changes in 
the fundamental physical theories, specifically quantum mechanics and quantum field theory. Inevitably a fundamental length should be involved in these theories[14-17]. This idea has been first suggested by a string theory[11]. But it is still considered to be a tentative theory without the experimental status and merely an attractive model. However, the fundamental length has been involved subsequently in more simple and natural considerations[12].

The main approach to framing of Quantum Mechanics with fundamental length (QMFL) and Quantum Field Theory with fundamental length (QFTFL) (or with Ultraviolet (UV) cutoff) is that associated with the Generalized Uncertainty Principle (GUP)[11-18]:

$$
\Delta x \geq \frac{\hbar}{\Delta p}+\alpha^{\prime} l_{p}^{2} \frac{\Delta p}{\hbar}
$$

with the corresponding Heisenberg algebra deformation produced by this principle[16-18].

Besides, in the works by the author[19-28] an approach to the construction of QMFL has been developed with the help of the deformed density matrix, the density matrix deformation in QMFL being a starting object called the density pro-matrix $\rho(\alpha) 3$ and deformation parameter (additional parameter)

$$
\alpha=l_{\min }^{2} / x^{2}
$$

where $x$ is the measuring scale, $l_{\min } \sim l_{p}$ and $0<\alpha \leq 1 / 4$ [19-25].

The explicit form of the above-mentioned deformation gives the exponential ansatz

$$
\rho^{*}(\alpha)=\exp (-\alpha) \sum_{i} \omega_{i}|i><i|
$$

where all $\omega_{i}>0$ are independent of $\alpha$ and their sum is equal to 1 .

In the corresponding deformed Quantum Theory (denoted as $Q F T^{\alpha}$ ) for average values we have

$$
<B>_{\alpha}=\exp (-\alpha)<B>
$$

where $\langle B\rangle$ - average in well-known QFT[24,25]. All the variables associated with the considered $\alpha$ - deformed quantum field theory are hereinafter marked with the upper index ${ }^{\alpha}$.

Note that the deformation parameter $\alpha$ is absolutely naturally represented as a ratio between the squared UV and IR limits

$$
\alpha=\left(\frac{U V}{I R}\right)^{2}
$$

where UV is fixed and IR is varying.

It should be noted[29] that in a series of the author's works[19-28] a minimal $\alpha$-deformation of QFT has been formed. By "minimal" it is meant that no space-time noncommutativity was required, i.e. there was no requirement for noncommutative operators associated with different spatial coordinates

$$
\left[X_{i}, X_{j}\right] \neq 0, i \neq j
$$

However, all the well-known deformations of QFT associated with GUP (for example[16-18]) contain (11) as an element of the corresponding deformed Heisenberg algebra. Because of this, it is necessary to extend (or modify) the above-mentioned minimal $\alpha$-deformation of QFT $-Q F T^{\alpha}$ [19-28] to some new deformation $\widetilde{Q F T}^{\alpha}$ compatible with GUP, as it has been noted in[29]. We can easily show that QFT parameter of deformations associated with GUP may be expressed in terms of the parameter $\alpha$ that has been introduced in the approach associated with the density matrix deformation[30,31]. Here the notation of[42] is used. Then

and

$$
[\vec{x}, \vec{p}]=i \hbar\left(1+\beta^{2} \vec{p}^{2}+\ldots\right)
$$

$$
\Delta x_{\min } \approx \hbar \sqrt{\beta} \sim l_{p}
$$

Then from (12), (13) it follows that $\beta \sim 1 / \mathbf{p}^{2}$, and for $x_{\min } \sim l_{p}, \beta$ corresponding to $x_{\min }$ is nothing else but

$$
\beta \sim 1 / P_{p l}^{2}
$$

where $P_{p l}$ is Planck's momentum: $P_{p l}=\hbar / l_{p}$.

In this way $\beta$ is changing over the following interval:

$$
\lambda / P_{p l}^{2} \leq \beta<\infty
$$

where $\lambda$ is a numerical factor and the second member in (12) is accurately reproduced in momentum representation (up to the numerical factor) by

$$
\begin{gathered}
\alpha=l_{\text {min }}^{2} / l^{2} \sim l_{p}^{2} / l^{2}=p^{2} / P_{p l}^{2} \\
{[\vec{x}, \vec{p}]=i \hbar\left(1+\beta^{2} \vec{p}^{2}+\ldots\right)==i \hbar\left(1+a_{1} \alpha+a_{2} \alpha^{2}+\ldots\right)}
\end{gathered}
$$

\section{Dynamical Cosmological Term and Its Modern Value}

As is known, a direct calculation of the vacuum energy density $\rho_{v a c}$ results in the value that is higher than the experimental one by a factor of $10^{122}$. According to the typical reasoning that is already "canonical" we have $[5,6,43]$ :

$$
<\rho_{\text {vac }}>=2 \int_{0}^{p_{\max }} d p \frac{4 \pi p^{2}}{(2 \pi \hbar)^{3}} \frac{\hbar \omega}{2}
$$

where $p_{\max } \sim P_{p l}$ is the momentum cut-off at Planck scales, because the General Relativity is valid right up to the Planck scales. Proceeding from $p=(\hbar \omega / c)$, we can obtain[43]

$$
<\rho_{v a c}>=\frac{\hbar \Omega^{4}}{8 \pi^{2} c^{3}}
$$

And then $\Omega$ is given by $\Omega_{p}$, where

$$
\hbar \Omega_{p}=E_{p}=\left(\frac{\hbar c^{5}}{G}\right)^{1 / 2}
$$

But it is known that $\left\langle\rho_{p}\right\rangle$ is higher than the observable value $\rho_{\text {vac }}^{\text {obs }}[6,43]$ approximately by a factor of $10^{122}$.

Yet, taking the cosmological term as a dynamic quantity, we can write (17) as

$$
<\rho_{v a c}(t)>=2 \int_{0}^{p(t)} d p \frac{4 \pi p^{2}}{(2 \pi \hbar)^{3}} \frac{\hbar \omega}{2}
$$


that in the very early Universe, at the time close to the Planck's time $t_{p}$, turns to (17) as follows:

$$
<\rho_{v a c}\left(t_{p}\right)>=2 \int_{0}^{P_{p l}} d p \frac{4 \pi p^{2}}{(2 \pi \hbar)^{3}} \frac{\hbar \omega}{2}
$$

However, it is known that the experimental value $\rho_{\text {vac }}^{\text {obs }}$ is sooner determined by low momenta and energies (corresponding to large scales) and also by the infrared limit that is given by a radius of the measurable part of the Universe $R_{\text {Univ }}=10^{28} \mathrm{~cm}$. For such momenta by the uncertainty principle (17) we have

$$
<\rho_{\text {vac }}\left(t_{\text {Univ }}\right)>=2 \int_{0}^{p_{\min }} d p \frac{4 \pi p^{2}}{(2 \pi \hbar)^{3}} \frac{\hbar \omega}{2}
$$

where $t_{\text {Univ }}$-Universe life-time, $p_{\min }$-"minimal" momentum.

As $R_{\text {Univ }} / l_{p}=P_{p l} / p_{\text {min }}=10^{61}$, then by analogy (18)and in the infrared limit

$$
<\rho_{\text {vac }}\left(t_{\text {Univ }}\right)>=\frac{\hbar \Omega_{\text {min }}^{4}}{8 \pi^{2} c^{3}}
$$

where

$$
\hbar \Omega_{\text {min }}=E_{\text {min }}
$$

But in this case $\rho_{\text {vac }}\left(t_{\text {Univ }}\right)$ is lower than $\rho_{\text {vac }}$ calculated from(17) by a factor of $10^{244}$ rather than by a factor of $10^{122}$.

This result is easily obtained using the Uncertainty Principle for the pair of conjugate variables $(\Lambda, V),[44-47]$ :

$$
\Delta V \approx \frac{\hbar}{\Delta \Lambda}
$$

where $\Lambda$ is the dynamic cosmological term, $V$ is the space-time volume. And $V$ results from the Einstein- Hilbert action $S_{E H}[45]$ :

$$
\Lambda \int d^{4} x \sqrt{-g}=\Lambda V
$$

where (26) is the term in $S_{E H}$.

$\operatorname{In}[30,31]$ the Uncertainty Principle (25) has been generalized at Planck scales up to the Generalized Uncertainty Principle

$$
\Delta V \approx \frac{\hbar}{\Delta \Lambda}+\alpha^{\prime} t_{p}^{2} \bar{V}^{2} \frac{\Delta \Lambda}{\hbar}
$$

where $\bar{V}$ - spatial part $V$ that, as is assumed, may be extracted explicitly.

(27) is of interest from the viewpoint of two limits[30,31]:

1) IR - limit: $t \rightarrow \infty$

2) UV - limit: $t \rightarrow t_{\text {min }}$.

In the case of IR-limit we have large volumes $\bar{V}$ and $V$ at low $\Delta \Lambda$. Because of this, the main contribution on the right-hand side of (27) is made by the first term, as great $\bar{V}$ in the second term is damped by small $t_{p}$ and $\Delta \Lambda$. Thus, we derive at

$$
\lim _{t \rightarrow \infty} \Delta V \approx \frac{\hbar}{\Delta \Lambda}
$$

in accordance with (25) and $\Lambda$ is a dynamic value fluctuating around zero.

And for the case 2) $\Delta \Lambda$ becomes significant

$$
\lim _{t \rightarrow t_{\min }} \bar{V}=\bar{V}_{\text {min }} \sim \bar{V}_{p}=l_{p}^{3} ; \lim _{t \rightarrow t_{\min }} V=V_{\min } \sim V_{p}=l_{p}^{3} t_{p}
$$

As a result, we have

$$
\lim _{t \rightarrow t_{\min }} \Delta V=\frac{\hbar}{\Delta \Lambda}+\alpha_{\Lambda} V_{p}^{2} \frac{\Delta \Lambda}{\hbar}
$$

where the parameter $\alpha_{\Lambda}$ absorbs all the above-mentioned proportionality coefficients.

For (30), $\Delta \Lambda \sim \Lambda_{p} \equiv \hbar / V_{p}=E_{p} / \bar{V}_{p}$.

It is easily seen that in this case $\Lambda \sim m_{p}^{4}$, in agreement with the value obtained using a standard (i.e. without super-symmetry and the like) quantum field theory[6,5]. Despite the fact that $\Lambda$ at Planck's scales (referred to as $\Lambda_{U V}$ ) (30) is also a dynamic quantity, it is not directly related to well-known $\Lambda$ (25),(28) (called $\Lambda_{I R}$ ) because the latter, as opposed to the first one, is derived from Einstein's equations

$$
R_{\mu v}-\frac{1}{2} g_{\mu v} R=8 \pi G_{N}\left(-\Lambda g_{\mu v}+T_{\mu v}\right)
$$

However, Einstein's equations (31) are not valid at the Planck scales and hence $\Lambda_{U V}$ may be considered as some high-energy generalization of the conventional cosmological constant, leading to $\Lambda_{I R}$ in the low-energy limit.

As $V \sim t l^{3} \sim l^{4}$, where $l$-characteristic linear dimension $V$, it is directly inferred that in the infrared region $\Lambda_{I R}$ calculated for $l=R_{\text {Univ }}$ is lower than $\Lambda_{U V}$ in $\sim R_{U n i v}^{4} / l_{p}^{4}=10^{244}$, that is in a perfect agreement with the result obtained by the direct calculations at the beginning of this Section.

In other words, a quantum field theory with UV-cutoff in the assumption that $\Lambda$ is a dynamic quantity gives no correct value for $\Lambda_{\text {observ }}$ as well.

In this way, similar to[48,49], we obtain

$$
\Lambda_{D E}=\sqrt{\Lambda_{U R} \Lambda_{I R}}
$$

where $\Lambda_{D E}=\Lambda_{\text {observ }}-$ cosmological term corresponding to the observable value.

Now we demonstrate that the Holographic Principle[50-53] is helpful in the solution of this problem, the deformation parameter $\alpha$ (7)"testing" variations of $\rho_{v a c}$ in time and hence playing the critical role.

In terms of the deformation parameter $\alpha$, the principal values of the Vacuum Energy Problem may be simply and clearly defined. Let us begin with the Schwarzschild black holes, whose semiclassical entropy is given by

$$
S=\pi R_{S c h}^{2} / l_{p}^{2}=\pi R_{S c h}^{2} m_{p}^{2}=\pi \alpha_{R_{S c h}}^{-1}
$$

with the assumption that in the formula for $\alpha R_{S c h}=x$ is the measuring scale and $l_{p}=1 / m_{p}$. Here $R_{S c h}$ is the adequate Schwarzschild radius, and $\alpha_{R_{S c h}}$ is the value of $\alpha$ associated with this radius. Then, as it has been pointed out in [55], in case the Fischler - Susskind cosmic holographic conjecture [56] is valid, the entropy of the Universe is limited by its "surface" measured in Planck units[55]:

$$
S \leq \frac{A}{4} m_{p}^{2}
$$

where the surface area $A=4 \pi R^{2}$ is defined in terms of the apparent (Hubble) horizon 


$$
R=\frac{1}{\sqrt{H^{2}+k / a^{2}}}
$$

with curvature $k$ and scale $a$ factors.

Again, interpreting $R$ from (35) as a measuring scale, we directly obtain(34) in terms of $a$ :

$$
S \leq \pi \alpha_{R}^{-1}
$$

where $\alpha_{R}=l_{p}^{2} / R^{2}$. Therefore, the average entropy density may be found as

$$
\frac{S}{V} \leq \frac{\pi \alpha_{R}^{-1}}{V}
$$

Using further the reasoning line of [55] based on the results of the holographic thermodynamics, we can relate the entropy and energy of a holographic system[54]. Similarly, in terms of the $\alpha$ parameter one can easily estimate the upper limit for the energy density of the Universe (denoted here by $\left.\rho_{\text {hol }}\right)$ :

$$
\rho_{\text {hol }} \leq \frac{3}{8 \pi R^{2}} m_{p}^{2}=\frac{3}{8 \pi} \alpha_{R} m_{p}^{4}
$$

that is drastically differing from the one obtained with well-known QFT

$$
\rho^{Q F T} \sim m_{p}^{4}
$$

Here by $\rho^{O F T}$ we denote the energy vacuum density calculated from well-known QFT (without UV cutoff)[5]. Obviously, as $\alpha_{R}$ for $R$ determined by (35) is very small, actually approximating zero, $\rho_{h o l}$ is by several orders of magnitude smaller than the value expected in QFT $-\rho^{Q F T}$.

Since $m_{p} \sim 1 / l_{p}$, the right-hand side of (38) is actually nothing else but the energy density in Holographic Dark Energy Models[57-60]. Thus, in Holographic Dark Energy Models the principal quantity, holographic energy density $\rho_{\Lambda}$, may be estimated in terms of the deformation parameter $\alpha$.

In fact, the upper limit of the right-hand side of (38) is attainable, as it has been indicated in[55]. The "overestimation" value of $r$ for the energy density $\rho^{Q F T}$, compared to $\rho_{\text {hol }}$, may be determined as

$$
r=\frac{\rho^{Q F T}}{\rho_{\text {hol }}}=\frac{8 \pi}{3} \alpha_{\mathbf{R}}^{-1}=\frac{8 \pi}{3} \frac{R^{2}}{l_{p}^{2}}=\frac{8 \pi}{3} \frac{S}{S_{p}}
$$

where $S_{p}$ is the entropy of the Plank mass and length for the Schwarzschild black hole. It is clear that due to smallness of $\alpha_{R}$ the value of $\alpha_{R}^{-1}$ is on the contrary too large. It may be easily calculated (e.g., see[55])

$$
r=5.44 \times 10^{122}
$$

in a good agreement with the astrophysical data.

Naturally, on the assumption that the vacuum energy density $\rho_{v a c}$ is involved in $\rho$ as a term

$$
\rho=\rho_{M}+\rho_{\text {vac }}
$$

where $\rho_{M}$ - average matter density, in case of $\rho_{v a c}$ we can arrive to the same upper limit (right-hand side of the formula (38)) as for $\rho$.
Average values of any quantity in QFT with UV-cutoff may be expended in terms of the small parameter $\alpha$ (Section 3$)$. Specifically, for the vacuum energy density $\rho(t)$ in the most general case we have

$$
<\rho(t)>=a_{0}+a_{1} \alpha(t)+a_{2} \alpha^{2}(t)+
$$

In[48,49] a similar expansion was performed for $\alpha(t)=\alpha_{I R}:$

$$
\rho_{\mathrm{vac}}=\frac{1}{l_{p}^{4}}+\frac{1}{l_{p}^{4}}\left(\frac{l_{p}}{l_{\Lambda}}\right)^{2}+\frac{1}{l_{p}^{4}}\left(\frac{l_{p}}{l_{\Lambda}}\right)^{4}+\ldots=\frac{1}{l_{p}^{4}}\left(1+\alpha_{I R}+\alpha_{I R}^{2}+\ldots\right)(44)
$$

Here $\rho_{\text {vac }}$ is understood as the quantity calculated for $l_{\Lambda}=l_{I R}=R_{\text {Univ }}$ and $\alpha_{I R}$ corresponds to $l_{I R}$.

But as shown previously, if the holographic principle is valid, this expansion has a more exact form

$$
<\rho(t)>=a_{1} \alpha(t)+a_{2} \alpha^{2}(t)+\ldots
$$

and $a_{1} \neq 0$.

\section{Gravitational Thermodynamics in Low and High Energy and Deformed Quantum Theory}

In the last decade a number of very interesting works have been published. We can primary name the works[61-70], where gravitation, at least for the в spaces with horizon, is directly associated with thermodynamics and the results obtained demonstrate a holographic character of gravitation. Of the greatest significance is a pioneer work[54]. For black holes the association has been first revealed in[71,72], where related the black-hole event horizon temperature to the surface gravitation. $\operatorname{In}[69]$, has shown that this relation is not accidental and may be generalized for the spaces with horizon. As all the foregoing results have been obtained in a semiclassical approximation, i.e. for sufficiently low energies, the problem arises: how these results are modified when going to higher energies. In the context of this paper, the problem may be stated as follows: since we have some infrared (IR) cutoff $l_{\max }$ and ultraviolet (UV) cutoff $l_{\min }$, we naturally have a problem how the above-mentioned results on Gravitational Thermodynamics are changed for

$$
l \rightarrow l_{\text {min }}
$$

Sections 2 and 3 of this paper show that the results are dependent on the deformation parameter $\alpha$ (33), (10) that in the accepted notation is of the form

$$
\alpha=\frac{l_{\min }^{2}}{l^{2}}
$$

In fact, in several papers[73-80] it has been demonstrated that thermodynamics and statistical mechanics of black holes in the presence of GUP (i.e. at high energies) should be modified. To illustrate, in[78] the Hawking temperature modification has been computed in the asymptotically flat space in this case in particular. It is easily seen that in this case the deformation parameter $\alpha$ arises naturally. Indeed, modification of the Hawking temperature is of the following 
form[73,75,78]:

$$
T_{G U P}=\left(\frac{d-3}{4 \pi}\right) \frac{\hbar r_{+}}{2 \alpha^{\prime 2} l_{p}^{2}}\left[1-\left(1-\frac{4 \alpha^{\prime 2} l_{p}^{2}}{r_{+}^{2}}\right)^{1 / 2}\right]
$$

where $d$ is the space-time dimension, and $r_{+}$is the uncertainty in the emitted particle position by the Hawking effect, expressed as

$$
\Delta x_{i} \approx r_{+}
$$

and being nothing else but a radius of the event horizon; $\alpha^{\prime}-$ dimensionless constant from GUP. But as we have $2 \alpha^{\prime} l_{p}=l_{\text {min }}$, in terms of $\alpha(48)$ may be written in a natural way as follows:

$$
T_{G U P}=\left(\frac{d-3}{4 \pi}\right) \frac{\hbar \alpha_{r_{+}}^{-1}}{\alpha^{\prime} l_{p}}\left[1-\left(1-\alpha_{r_{+}}\right)^{1 / 2}\right]
$$

where $\alpha_{r_{+}}$- parameter $\alpha$ associated with the IR-cutoff $r_{+}$. In such a manner $T_{G U P}$ is only dependent on the constants including the fundamental ones and on the deformation parameter $\alpha$.

The dependence of the black hole entropy on $\alpha$ may be derived in a similar way. For a semiclassical approximation of the Bekenstein-Hawking formula[71,72]

$$
S=\frac{1}{4} \frac{A}{l_{p}^{2}}
$$

where $A$ - surface area of the event horizon, provided the horizon event has radius $r_{+}$, then $A \sim r_{+}^{2}$ and (51) is clearly of the form

$$
S=\sigma \alpha_{r_{+}}^{-1}
$$

where $\sigma$ is some dimensionless denumerable factor. The general formula for quantum corrections[77] given as

$$
S_{G U P}=\frac{A}{4 l_{p}^{2}}-\frac{\pi \alpha^{\prime 2}}{4} \ln \left(\frac{A}{4 l_{p}^{2}}\right)++\sum_{n=1}^{\infty} c_{n}\left(\frac{A}{4 l_{p}^{2}}\right)^{-n}+\text { const }
$$

where the expansion coefficients $c_{n} \propto \alpha^{2(n+1)}$ can always be computed to any desired order of accuracy[77], may be also written as a power series in $\alpha_{r_{+}}^{-1}$ (or Laurent series in $\left.\alpha_{r_{+}}\right)$

$$
S_{G U P}=\sigma \alpha_{r_{+}}^{-1}-\frac{\pi \alpha^{\prime 2}}{4} \ln \left(\sigma \alpha_{r_{+}}^{-1}\right)+\sum_{n=1}^{\infty}\left(c_{n} \sigma^{-n}\right) \alpha_{r_{+}}^{n}+\text { const }
$$

Note that here no consideration is given to the restrictions on the IR-cutoff

$$
l \leq l_{\max }
$$

and to those corresponding the extended uncertainty principle (EUP)[78] or symmetric generalized uncertainty principle (SGUP)[79] that leads to a minimal momentum.

A black hole is a specific example of the space with horizon. It is clear that for other horizon spaces[69] a similar relationship between their thermodynamics and the deformation parameter $\alpha$ should be exhibited.

Quite recently, in a series of papers, and specifically in[61-67], it has been shown that Einstein equations may be derived from the surface term of the GR Lagrangian, in fact containing the same information as the bulk term.
It should be noted that Einstein's equations[at least for space with horizon] may be obtained from the proportionality of the entropy and horizon area together with the fundamental thermodynamic relation connecting heat, entropy, and temperature[54]. In fact[61-68], this approach has been extended and complemented by the demonstration of holographicity for the gravitational action (see also[69]). And in the case of Einstein-Hilbert gravity, it is possible to interpret Einstein's equations as the thermodynamic identity[70]:

$$
T d S=d E+P d V
$$

The above-mentioned results have been obtained at low energies, i.e. in a semiclassical approximation. Because of this, the problem arises how these results are changed in the case of high energies? Or more precisely, how the results of $[54,61-70]$ are generalized in the UV-limit? It is obvious that, as in this case all the thermodynamic characteristics become dependent on the deformation parameter $\alpha$, all the corresponding results should be modified (deformed) to meet the following requirements:

(a) to be clearly dependent on the deformation parameter $\alpha$ at high energies;

(b) to be duplicated, with high precision, at low energies due to the suitable limiting transition.

The problem may be more specific. As, according to[54, $69,70]$ and some other works, gravitation is greatly determined by thermodynamics, and at high energies the latter is a "deformation of the classical thermodynamics". Here "highenergy deformation of thermodynamics" is understood as some (meanwhile unknown) deformation of thermodynamics in high energies. This theory is still unframed, though several of its elements $T_{G U P}, S_{G U P}$ and the like[73-80] are known already. It is interesting whether gravitation at high energies (or what is the same, quantum gravity or Planck scale) is being determined by the corresponding deformed thermodynamics. The formulae (50) and (54) are elements of the high-energy $\alpha$-deformation in thermodynamics, a general pattern of which still remains to be formed. Obviously, these formulae should be involved in the general pattern giving better insight into the quantum gravity, as they are applicable to black mini-holes (Planck black holes) which may be a significant element of such a pattern. But what about other elements of this pattern? How can we generalize the results $[54,69,70]$ when the IR-cutoff tends to the UVcutoff (formula (46))? What are modifications of the thermodynamic identity (56) in a high-energy deformed thermodynamics and how is it applied in high-energy (quantum) gravity?

By authors opinion, the methods developed to solve the problem of point (c) and elucidation of other above- mentioned problems may form the basis for a new approach to solution of the quantum gravity problem. And one of the keys to the quantum gravity problem is a better insight into the high-energy thermodynamics.

\section{6. $\alpha$-Representation of Einstein's Equations}


Let us consider $\alpha$-representation and high energy $\alpha$ -deformation of the Einstein's field equations for the specific cases of horizon spaces (the point (c) of Section 4). In so doing the results of the survey work[81] are used. Then, specifically, for a static, spherically symmetric horizon in space-time described by the metric

$$
d s^{2}=-f(r) c^{2} d t^{2}+f^{-1}(r) d r^{2}+r^{2} d \Omega^{2}
$$

the horizon location will be given by simple zero of the function $f(r)$, at $r=a$.

It is known that for horizon spaces one can introduce the temperature that can be identified with an analytic continuation to imaginary time. In the case under consideration ([81], eq.(116))

$$
k_{B} T=\frac{\hbar c f^{\prime}(a)}{4 \pi}
$$

Therewith, the condition $f(a)=0$ and $f^{\prime}(a) \neq 0$ must be fulfilled.

Then at the horizon $r=a$ Einstein's field equations

$$
\frac{c^{4}}{G}\left[\frac{1}{2} f^{\prime}(a) a-\frac{1}{2}\right]=4 \pi P a^{2}
$$

may be written as the thermodynamic identity $(56)([81]$ formula (119))

$$
\underbrace{\frac{\hbar c f^{\prime}(a)}{4 \pi}}_{k_{B} T} \underbrace{\frac{c^{3}}{G \hbar} d\left(\frac{1}{4} 4 \pi a^{2}\right)}_{d S} \underbrace{-\frac{1}{2} \frac{c^{4} d a}{G}}_{-d E}=\underbrace{P d\left(\frac{4 \pi}{3} a^{3}\right)}_{P d V}
$$

where $P=T_{r}^{r}$ is the trace of the momentum-energy tensor and radial pressure. In the last equation $d a$ arises in the infinitesimal consideration of Einstein's equations when studying two horizons distinguished by this infinitesimal quantity $a$ and $a+d a$ ([81] formula (118)).

Now we consider (60) in new notation expressing $a$ in terms of the corresponding deformation parameter $\alpha$. Then we have

$$
a=l_{\min } \alpha^{-1 / 2}
$$

Therefore,

$$
f^{\prime}(a)=-2 l_{\min }^{-1} \alpha^{3 / 2} f^{\prime}(\alpha)
$$

Substituting this into (59) or into (60), we obtain in the considered case of Einstein's equations in the " $\alpha$ - representation" the following:

$$
\frac{c^{4}}{G}\left(-\alpha f^{\prime}(\alpha)-\frac{1}{2}\right)=4 \pi P \alpha^{-1} l_{\text {min }}^{2}
$$

Multiplying the left- and right-hand sides of the last equation by $\alpha$, we get

$$
\frac{c^{4}}{G}\left(-\alpha^{2} f^{\prime}(\alpha)-\frac{1}{2} \alpha\right)=4 \pi P l_{\text {min }}^{2}
$$

But since usually $l_{\min } \sim l_{p}$ (that is just the case if the Generalized Uncertainty Principle (GUP) is satisfied), we have $l_{\min }^{2} \sim l_{p}^{2}=G \hbar / c^{3}$. When selecting a system of units, where $\hbar=c=1$, we arrive at $l_{\min } \sim l_{p}=\sqrt{ } G$, and then (63) is of the form

$$
-\alpha^{2} f^{\prime}(\alpha)-\frac{1}{2} \alpha=4 \pi P \vartheta^{2} G^{2}
$$

where $\vartheta=l_{\text {min }} / l_{p}$. L.h.s. of (65) is dependent on $\alpha$. Because of this, r.h.s. of (65) must be dependent on $\alpha$ as well, i. e. $P=P(\alpha)$.

Analysis of $\alpha$-Representation of Einstein's Equations Now let us get back to (60). In[81] the low-energy case has been considered, for which ([81] formula (120))

$$
S=\frac{1}{4 l_{p}^{2}}\left(4 \pi a^{2}\right)=\frac{1}{4} \frac{A_{H}}{l_{p}^{2}} ; E=\frac{c^{4}}{2 G} a=\frac{c^{4}}{G}\left(\frac{A_{H}}{16 \pi}\right)^{1 / 2}
$$

where $A_{H}$ is the horizon area. In our notation (66) may be rewritten as

$$
S=\frac{1}{4} \pi \alpha^{-1} ; E=\frac{c^{4}}{2 G} a=\frac{c^{4}}{G}\left(\frac{A_{H}}{16 \pi}\right)^{1 / 2}=\frac{\vartheta}{2 \sqrt{ } G} \alpha^{1 / 2}
$$

We proceed to two entirely different cases: low energy (LE) case and high energy (HE) case. In our notation these are respectively given by

A) $\alpha \rightarrow 0$ (LE), B) $\alpha \rightarrow 1 / 4$ (HE),

C) $\alpha$ complies with the familiar scales and energies.

The case of C) is of no particular importance as it may be considered within the scope of the conventional General Relativity.

Indeed, in point A) $\quad \alpha \rightarrow 0$ is not actually an exact limit as a real scale of the Universe (Infrared (IR) - cutoff $l_{\text {max }}=R_{\text {Univ }} \approx 10^{28} \mathrm{~cm}$ ), and then

$$
\alpha_{\min } \sim l_{p}^{2} / l_{\max }^{2} \approx 10^{-122} .
$$

In this way A) is replaced by A1) $\alpha \rightarrow \alpha_{\text {min }}$. In any case at low energies the second term in the left-hand side (65) may be neglected in the infrared limit. Consequently, at low energies (65) is written as

$$
-\alpha^{2} f^{\prime}(\alpha)=4 \pi P(\alpha) \vartheta^{2} G^{2}
$$

Solution of the corresponding Einstein equation - finding of the function $f(\alpha)=f[P(\alpha)]$ satisfying (68). In this case formulae (66) are valid as at low energies a semiclassical approximation is true. But from (68) it follows that

$$
f(\alpha)=-4 \pi \vartheta^{2} G^{2} \int \frac{P(\alpha)}{\alpha^{2}} d \alpha
$$

On the contrary, knowing $f(\alpha)$, we can obtain $P(\alpha)=T_{r}^{r}$.

But it is noteworthy that, when studying the infrared modified gravity[82-84], we have to make corrections for the considerations of point A1).

\section{Possible High Energy $\alpha$-Deformation of General Relativity}

Let us consider the high-energy case B). Here two variants are possible.

\section{First variant}

In this case it is assumed that in the high-energy (Ultraviolet (UV)) limit the thermodynamic identity (60) (or that is the same (56) is retained but now all the quantities involved in this identity become $\alpha$-deformed. This means that they 
appear in the $\alpha$-representation with quantum corrections and are considered at high values of the parameter $\alpha$, i.e. at $\alpha$ close to $1 / 4$. In particular, the temperature $T$ from equation (60) is changed by $T_{G U P}$ (50), the entropy $S$ from the same equation given by semiclassical formula (66) is changed by $S_{G U P}$ (54), and so forth:

$$
E \mapsto E_{G U P}, V \mapsto V_{G U P}
$$

Then the high-energy $\alpha$-deformation of equation (60) takes the form

$$
k_{B} T_{G U P}(\alpha) d S_{G U P}(\alpha)-d E_{G U P}(\alpha)=P(\alpha) d V_{G U P}(\alpha)
$$

Substituting into (70) the corresponding quantities

$T_{G U P}(\alpha), S_{G U P}(\alpha), E_{G U P}(\alpha), V_{G U P}(\alpha), P(\alpha)$ and expanding them into a Laurent series in terms of $\alpha$, close to high values of $\alpha$, specifically close to $\alpha=1 / 4$, we can derive a solution for the high energy $\alpha$-deformation of general relativity (70) as a function of $P(\alpha)$. As this takes place, provided at high energies the generalization of $(60)$ to $(70)$ is possible, we can have the high-energy $\alpha$-deformation of the metric. Actually, as from (60) it follows that

$$
f^{\prime}(a)=\frac{4 \pi k_{B}}{\hbar c} T=4 \pi k_{B} T
$$

(considering that we have assumed $\hbar=c=1$ ), we get

$$
f^{\prime}{ }_{G U P}(a)=4 \pi k_{B} T_{G U P}(\alpha)
$$

L.h.s. of (72) is directly obtained in the $\alpha$-representation. This means that, when $f^{\prime} \sim T$, we have $f_{G U P}^{\prime} \sim T_{G U P}$ with the same factor of proportionality. In this case the function $f_{G U P}$ determining the high-energy $\alpha$-deformation of the spherically symmetric metric may be in fact derived by the expansion of $T_{G U P}$, that is known from (50), into a Laurent series in terms of $\alpha$ close to high values of $\alpha$ (specifically close to $\alpha=1 / 4$ ), and by the subsequent integration.

It might be well to remark on the following.

7.1 As on going to high energies we use (GUP), $\vartheta$ from equation (65) is expressed in terms of $\alpha^{\prime}$-dimensionless constant from GUP (6), (50): $\vartheta=2 \alpha^{\prime}$.

7.2 Of course, in all the formulae including $l_{p}$ this quantity must be changed by $G^{1 / 2}$ and hence $l_{\min }$ by $\vartheta G^{1 / 2}=2 \alpha^{\prime} G^{1 / 2}$.

7.3 As noted in the end of subsection 6.1, and in this case also knowing all the high-energy deformed quantities $T_{G U P}(\alpha), S_{G U P}(\alpha), E_{G U P}(\alpha), V_{G U P}(\alpha)$, we can find $P(\alpha)$ at $\alpha$ close to $1 / 4$.

7.4 Here it is implicitly understood that the Ultraviolet limit of Einstein's equations is independent of the starting horizon space. This assumption is quite reasonable. Because of this, we use the well-known formulae for the modification of thermodynamics and statistical mechanics of black holes in the presence of GUP[73-80]

7.5 The use of the thermodynamic identity (70) for the description of the high energy deformation in General Relativity implies that on going to the UV-limit of Einstein's equations for horizon spaces in the thermodynamic representation (consideration) we are trying to remain within the scope of equilibrium statistical mechanics[85] (equilibrium thermodynamics)[86]. However, such an assumption seems to be too strong. But some grounds to think so may be found as well. Among other things, of interest is the result from[73] that GUP may prevent black holes from their total evaporation. In this case the Planck's remnants of black holes will be stable, and when they are considered, in some approximation the equilibrium thermodynamics should be valid. At the same time, by author's opinion these arguments are rather weak to think that the quantum gravitational effects in this context have been described only within the scope of equilibrium thermodynamics[86].

\section{Second variant}

According to the remark of .5, it is assumed that the interpretation of Einstein's equations as a thermodynamic identity (60) is not retained on going to high energies (UV-limit), i.e. at $\alpha \rightarrow 1 / 4$, and the situation is adequately described exclusively by non-equilibrium thermodynamics $[86,87]$. Naturally, the question arises: which of the additional terms introduced in (60) at high energies may be leading to such a description?

In the $[30,31]$ it has been shown that in case the cosmological term $\Lambda$ is a dynamic quantity, it is small at low energies and may be sufficiently large at high energies. In the right-hand side of (65) in the $\alpha$-representation the additional term $G(\Lambda(\alpha))$ is introduced:

$$
-\alpha^{2} f^{\prime}(\alpha)-\frac{1}{2} \alpha=4 \pi P(\alpha) \vartheta^{2} G^{2}-G \Lambda(\alpha)
$$

where $\Lambda(\alpha)$ is the cosmological term depending from $\alpha$. Then its inclusion in the low-energy case (59)(or in the $\alpha$ representation (65)) has actually no effect on the thermodynamic identity (60) validity, and consideration within the scope of equilibrium thermodynamics still holds true. It is well known that this is not the case at high energies as the $\Lambda$ -term may contribute significantly to make the "process" non-equilibrium in the end[86,87].

Is this the only cause for violation of the thermodynamic identity (60) as an interpretation of the high-energy generalization of Einstein's equations? Further investigations are required to answer this question.

\section{Conclusions}

Thus, this work presents an analysis of the dark energy problem on the assumption that it is the time-varying vacuum energy, that conforms to the dynamic cosmological term $\Lambda(t)$. Note that the question of the explicit relationship between $\Lambda$ and $t$ is not touched, we have rather studied the problem of deriving the currently observable value for $\Lambda=\Lambda_{\text {observ }}$.

As such a consideration inevitably involves the physics of the early Universe (Planck scales) and hence QFT with the fundamental length (on the order of Planck's $l_{p}$ ), it is associated with new parameters (deformation parameters) and, in the limiting transition to low-energy, QFT with the funda- 
mental length turns to well-known QFT (without cutoff). By the author's opinion, $\alpha$ (7) is the most optimal of the deformation parameters from the standpoint of the problems considered in this work.

The results obtained in Sections 6 and 7 enable framing of the following hypothesis:

a dynamic cosmological term is a measure of deviation from the thermodynamic identity (the first law of thermodynamics) of the high-energy (Planck's) deformation of Einstein equations for horizon spaces in their thermodynamic interpretation.

The dynamic cosmological term correlates well with inflation models[4] as the latter require a very high $\Lambda$ at the early stages of the Universe, and this is distinct from $\Lambda=\Lambda_{\text {exper }}$ in the modern period. Of great interest is the recent work[89], where a mechanism of the vacuum energy decay in the de Sitter space is established to support a dynamic nature of $\Lambda$.

This work is a step to the incorporation of deformation parameters involved in a quantum field theory at Planck's scales into the high-energy deformation of the General Relativity (GR). The corresponding calculations with the adequate interpretation must follow next. It is interesting to consider the high energy $\alpha$-deformation of GR in a more general case. The problem is how far a thermodynamic interpretation of Einstein's equations may be extended? We should remember that, as all the deformations considered involve a minimal length at the Planck level $l_{\min } \sim l_{p}$, a minimal three-dimensional volume should also be the case $V_{\text {min }} \sim V_{p}=l_{p}^{3}$. And this is of particular importance for high energy thermodynamics (some indications to this fact have been demonstrated in[30,31]).

\section{REFERENCES}

[1] Mukohyama, S., Randall, L. A, 2004, Dynamical approach to the cosmological constant., Phys.Rev.Lett., 92, 211302.; L.P. Chimento and D. Pavon, 1998, Inhomogeneous Universe Models with Varying Cosmological Term, Gen. Rel. Grav., 643--651; S. Carneiro,2003, Decaying Lambda cosmology with varying $\mathrm{G}$, arXiv:gr-qc/0307114

[2] Aldrovandi, R. Beltran Almeida, J. P., Pereira, J. G., 2005 , Time-Varying Cosmological Term: Emergence and Fate of a FRW Comments., Grav.Cosmol. 11, 277-283

[3] Richard T., 2008, Hammond, Terry Pilling, Dark Entropy, arXiv: 0806.1277

[4] Kolb E., and Turner M., 1990, The Early Universe, Reading, Addison Wesley; Baumann D.,2009, TASI Lectures on Inflation , arXiv:0907.5424

[5] Zel'dovich,Y.B., 1968, Sov.Phys.Uspehi, 11, 381-393

[6] Weinberg, S., 1989, the Cosmological Constant Problem. Rev. Mod. Phys., 61 , 1-23

[7] Klinkhamer F.R., 2007, Fundamental length scale of quantum spacetime foam., JETPLett., 86, 2167-2180

[8] Amelino-Camelia G. Smolin L., 2009, Prospects for constraining quantum gravity dispersion with near term observations., Phys.Rev.D ,80, 084017; Gubitosi G. et al., 2009, A Constraint on Planck-scale Modifications to Electrodynamics with CMB polarization data., JCAP,0908, 021; AmelinoCamelia G., 2005, Building a case for a Planck-scale- deformed boost action: the Planck-scale particle-localization limit., Int.J.Mod.Phys. D.,14, 2167-2180

[9] Hossenfelder S. et al.,2003, Signatures in the Planck Regime., Phys. Lett.B.,575, 85-99; Hossenfelder S., 2004, Running coupling with minimal length, Phys.Rev.D., 70, 105003; Hossenfelder S., 2006, Self-consistency in theories with a minimal length, Class. Quant. Grav.,23, 1815-1821

[10] Faddeev, L.,1989, Mathematical View on Evolution of Physics., Priroda, 5, 11-18

[11] Veneziano,G. A, 1986, Stringy Nature Needs Just Two Constants.,Europhys. Lett., 2,199-211; Amati, D., Ciafaloni, M., Veneziano,G.,1989, Can Space-Time Be Probed Below the String Size? Phys.Lett.B, 216,41-47; E.Witten, 1996. Phys. Today, 49, 24-28

[12] Adler,R.J., and Santiago,D.I., 1999, On gravity and the uncertainty principle., Mod. Phys. Lett. A ,14, 1371-1378

[13] Scardigli,F.,1999, Generalized uncertainty principle in quantum gravity from micro - black hole Gedanken experiment., Phys. Lett. B., 452, 39-44; Bambi,C. A, 2008, Revision of the Generalized Uncertainty Principle., Class. Quant. Grav, 25, 105003

[14] Garay, L.,1995. Quantum gravity and minimum length. Int.J. Mod.Phys.A, 10, 145-166

[15] Ahluwalia, D.V., 2000, Wave particle duality at the Planck scale: Freezing of neutrino oscillations., Phys.Lett, A., 275, 31-35; Ahluwalia,D.V., 2002, Interface of gravitational and quantum realms., Mod.Phys.Lett., A. 17, 1135-1146

[16] Maggiore, M. A., 1993, Generalized uncertainty principle in quantum gravity., Phys.Lett, 304, 65-69

[17] Maggiore, M.,1993, The Algebraic structure of the generalized uncertainty principle, Phys.Lett.B, 319, 83-86

[18] Kempf,A.; Mangano,G.; Mann,R.B.,1995, Hilbert space representation of the minimal length uncertainty relation. Phys.Rev.D, 52, 1108-1118

[19] Shalyt-Margolin,A.E., and Suarez, J.G.,2003, Quantum mechanics of the early universe and its limiting transition. gr-qc/0302119, 16pp

[20] Shalyt-Margolin, A.E. and Suarez, J.G., 2003, Quantum mechanics at Planck's scale and density matrix. Intern. Journ. Mod. Phys D. ,12, 1265-1278

[21] Shalyt-Margolin, A.E., and Tregubovich, A.Ya., 2004, Deformed density matrix and generalized uncertainty relation in thermodynamics., Mod. Phys.Lett. A., 19, 71-82

[22] Shalyt-Margolin, A.E., 2004, Nonunitary and unitary transitions in generalized quantum mechanics, new small parameter and information problem solving., Mod. Phys. Lett. A, 19, 391-404

[23] Shalyt-Margolin, A.E., 2004, Pure states, mixed states and 
Hawking problem in generalized quantum mechanics. Mod. Phys. Lett. A, 19, 2037-2045

[24] Shalyt-Margolin, A.E., 2004, The Universe as a nonuniform lattice in finite volume hypercube. I. Fundamental definitions and particular features., Intern. Journ. Mod.Phys D, 13, 853864

[25] Shalyt-Margolin, A.E., 2005, The Universe as a nonuniform lattice in the finite-dimensional hypercube. II. Simple cases of symmetry breakdown and restoration. Intern.Journ.Mod. Phys.A, 20, 4951-4964

[26] Shalyt-Margolin, A.E. and Strazhev, V.I., 2006, The Density Matrix Deformation in Quantum and Statistical Mechanics in Early Universe., Proc. Sixth International Symposium "Frontiers of Fundamental and Computational Physics", edited by B.G. Sidharth at al. Springer, 131-134

[27] Shalyt-Margolin, A.E., 2005, The Density matrix deformation in physics of the early universe and some of its implications., Quantum Cosmology Research Trends,edited by A. Reimer, Horizons in World Physics., Nova Science Publishers, Inc., Hauppauge, 49-91

[28] Shalyt-Margolin, A.E., 2006, Deformed density matrix and quantum entropy of the black hole. Entropy, 8, 31-43

[29] Shalyt-Margolin, A.E., 2007, Entropy in the Present and Early Universe, Symmetry, 18, 299-320

[30] Shalyt-Margolin, A.E., 2010, Entropy in the Present and Early Universe and Vacuum Energy, AIP Conference Proceedings, 1205, 160-167

[31] Shalyt-Margolin, A.E.,2010, Entropy In The Present And Early Universe: New Small Parameters And Dark Energy Problem, Entropy ,12, 932-952

[32] Sciama, D.W., 1984, Proc. R.Soc.Lond. A 394, 1-23; Bowyer S., KorpelaE.J., Edelstein J., Lampton M., Morales C., Perez-Mercader J., Gomez J.F., Trapero J., 1999, ApJ 526, 10--31, Turner, M.S., 1991, Phys. scr.,36, 167--179

[33] Perlmutter, S. et al., 1999, Measurements of Omega and Lambda from 42 high redshift supernovae., Astrophys. J ,517, 565-586; Riess A. G. et al.,1998, Observational evidence from supernovae for an accelerating universe and a cosmological constant., Astron. J. ,116, 1009-1038; Riess A. G. et al., 1999, BV RI light curves for 22 type Ia supernovae. Astron. J , 117, 707-724

[34] Dan Hooper, 2009, TASI 2008 Lectures on Dark Matter, ArXiv: 0901.4090

[35] Ratra,B. and Peebles,J.,1988,Cosmological Consequences of a Rolling Homogeneous Scalar Field., Phys. Rev. D,37, 3406-3422; Caldwell,R. R., Dave, R. and Steinhardt, P. J. Cosmological imprint of an energy component with general equation of state., 1998, Phys. Rev. Lett , 80, 1582-1585

[36] Armendariz-Picaon, C., Damour,T.V., Mukhanov, V., 1999, k - inflation., Phys. Lett. B, 458, 209-218; J. Garriga, and V. Mukhanov, Perturbations in k-inflation., Phys. Lett. B,458, 219-225

[37] Padmanabhan, T., 2002, Accelerated expansion of the universe driven by tachyonic matter. Phys. Rev. D,66, 021301; Bagla, J. S. Jassal,H. K., Padmanabhan, T.,2003, Cosmology with tachyon field as dark energy., Phys. Rev. D, 67,063504; Abramo, L. R. W., and Finelli,F.,2003, Cosmological dy- namics of the tachyon with an inverse power-law potential., Phys. Lett. B , 575, 165-171

[38] Sahni, V. and Shtanov, Y., 2003, Brane world models of dark energy., JCAP,0311, 014--027; Elizalde, E., Nojiri, S., Odintsov, S. D.,2004, Late-time cosmology in (phantom) scalar-tensor theory: Dark energy and the cosmic speed-up. Phys. Rev. D ,70, 043539

[39] Gliner, E. B.,1965, Algebraic Properties of Energy- Momentum Tensor and vacuum-like state of the matter, ZHETF, 49, 542-549

[40] Wald, Robert. M., 1984, General Relativity. The University Chicago Press., Chicago and London 1984, 491 p.p

[41] Chakraborty, W.,2011, Acceleration Expantion of the Universe, ArXiv: 1105.1087

[42] Kim,Yong-Wan, Lee,Hyung Won, Myung, Yun Soo,2009, Entropy bound of local quantum field theory with generalized uncertainty principle., Phys.Lett.B, 673,293-296

[43] O'Connell, R. F. ,2007, Phys.Lett.A, 366:177-178

[44] Jejjala, V., Kavic, M., Minic, D., 2007, Time and M-theory. Int. J. Mod. Phys. A ,22, 3317-3405

[45] Jejjala, V., Kavic, M., Minic, D.,2007, Fine structure of dark energy and new physics., Adv. High Energy Phys, 2007, 21586

[46] Jejjala, V. and Minic, D.,2007, Why there is something so close to nothing: Towards a fundamental theory of the cosmological constant., Int.J.Mod.Phys.A ,22, 1797-1818

[47] Jejjala, V., Minic, D.,Tze, C-H., 2004, Toward a background independent quantum theory of gravity. Int. J. Mod. Phys. D, $13,2307-2314$

[48] Padmanabhan, T., 2005, Darker side of the universe. and the crying need for some bright ideas! Proceedings of the 29th International Cosmic Ray Conference, Pune, India, 47-62.

[49] Padmanabhan, T., 2006, Dark Energy: Mystery of the Millennium. Paris 2005, Albert Einstein's century, AIP Conference Proceedings 861, American Institute of Physics, New York, 858-866

[50] Hooft, G. 'T.,1993, Dimensional reduction in quantum gravity.Essay dedicated to Abdus Salam gr-qc/9310026, 15pp

[51] Hooft, G. 'T., 2000, The Holographic Principle, hep-th/ 0003004, 15pp.; L.Susskind, 1995, The World as a hologram. J. Math. Phys, 36, 6377-6396

[52] Bousso, R. The Holographic principle, 2002, Rev. Mod. Phys, $74,825-874$

[53] Bousso, R. A, 1999, Covariant entropy conjecture. JHEP ,007, 004--021

[54] Jacobson, T.,1995, Thermodynamics of space-time: The Einstein equation of state, Phys. Rev. Lett ,75, 1260-1263

[55] Balazs,C. and Szapudi,I.,2006, Naturalness of the vacuum energy in holographic theories., hep-th/0603133, 4pp

[56] Fischler,W. and Susskind, L.,1998, Holography and cosmology. hep-th/9806039, 7pp

[57] Cohen,A., Kaplan, D., Nelson, A.,1999, Effective field theory, 
black holes, and the cosmological constant., Phys. Rev. Lett ,82, 4971-4974

[58] Myung,Y.S., 2005,Holographic principle and dark energy. Phys. Lett. B,610, 18-22

[59] Myung, Y. S. and Min-Gyun Seo, 2009, Origin of holographic dark energy models., Phys. Lett. B,617, 435-439

[60] Sergio del Campo, Júlio.C. Fabris, Ramón Herrera, Winfried Zimdahl,2011, On holographic dark-energy models, Phys. Rev.D, 83, 123006

[61] Padmanabhan, T.,2005, A New perspective on gravity and the dynamics of spacetime. Int.Jorn.Mod.Phys. D, 14, 2263 2270

[62] Padmanabhan, T., 2002, The Holography of gravity encoded in a relation between entropy, horizon area and action for gravity., Gen.Rel.Grav , 34, 2029-2035

[63] Padmanabhan,T.,2005, Holographic Gravity and the Surface term in the Einstein-Hilbert Action., Braz.J.Phys, 35, $362-372$

[64] Padmanabhan, T., 2006, Gravity: A New holographic perspective. Int.J.Mod.Phys.D, 15, 1659-1676

[65] Mukhopadhyay,A. and Padmanabhan,T.,2006, Holography of gravitational action functionals, Phys.Rev.D, 74, 124023

[66] Padmanabhan, T., 2008, Dark energy and gravity, Gen.Rel. Grav, 40, 529-564

[67] Padmanabhan,T. and Paranjape,A.,2007, Entropy of null surfaces and dynamics of spacetime, Phys.Rev.D.,75, 064004

[68] Padmanabhan, T., 2007, Gravity as an emergent phenomenon: A conceptual description. International Workshop and at on Theoretical High Energy Physics (IWTHEP 2007), AIP Conference Proceedings 939, American Institute of Physics, New York, 114-123

[69] Padmanabhan, T., 2005, Gravity and the thermodynamics of horizons, Phys.Rept , 406, 49-125

[70] Paranjape, A., Sarkar, S.; Padmanabhan, T.,2006, Thermodynamic route to field equations in Lancos- Lovelock gravity, Phys.Rev. D, 74, 104015

[71] Bekenstein, J.D., 1973, Black Holes and Entropy. Phys. Rev. D.7, 2333-2345

[72] Hawking, S., 1976, Black Holes and Thermodynamics, Phys. Rev.D, 13,191-204

[73] Adler, R. J., Chen,P., Santiago, D. I.,2001, The generalized uncertainty principle and black hole remnants, Gen.Rel. Grav., 13, 2101-2108
[74] Custodio, P. S. and Horvath, J. E.,2003, The Generalized uncertainty principle, entropy bounds and black hole (non)evaporation in a thermal bath, Class.Quant.Grav.,20, L197-L203

[75] Cavaglia, M. and Das, S., 2004, How classical are TeV scale black holes? Class.Quant.Grav., 21, 4511-4523

[76] Bolen, B., and Cavaglia,M., 2005,(Anti-)de Sitter black hole thermodynamics and the generalized uncertainty principle., Gen.Rel.Grav.,37， 1255-1263

[77] Medved, A.J.M. and Vagenas, E.C., 2004, When conceptual worlds collide: The GUP and the BH entropy. Phys. Rev. D ,70, 124021

[78] Park, M.-I.,2008, The Generalized Uncertainty Principle in (A)dS Space and the Modification of Hawking Temperature from the Minimal Length., Phys.Lett.B , 659, 698-702

[79] Kim,Wontae. and Son,Edwin J., 2008, Yoon, Myungseok. Thermodynamics of a black hole based on a generalized uncertainty principle. JHEP,08, 035--043

[80] Nouicer,K,2007,Quantum-corrected black hole thermodynamics to all orders in the Planck length, Phys.Lett B,647, 63--71

[81] Padmanabhan,T.,2010, Thermodynamical Aspects of Gravity: New insights.Rep. Prog. Phys. 73, 046901

[82] Patil, S.P., 009, Degravitation, Inflation and the Cosmological Constant as an Afterglow. JCAP,0901, 017--035

[83] Park, Mu-in., 2009, The Black Hole and Cosmological Solutions in IR modified Horava Gravity. JHEP,0909, 123--142

[84] Rubakov, V. A., and Tinyakov,P. G., 2008, Infrared-modified gravities and massive gravitons. Phys.Usp, 123., 759-792; Nikiforova,V.; Randjbar-Daemi, S.; Rubakov V., 2009, Infrared Modified Gravity with Dynamical Torsion. Phys. Rev. D , 80, 124050

[85] Balesku, R.,1975 Equilibruim and Nonequilibruim Statistical Mechanics, v.1, A Wiley Interscience Publications, New York-London-Sydney-Toronto

[86] Bazarov, I.P., 1991, Thermodynamics, Moskow, Press "Higher School"

[87] Gyarmati, I., 1974, Non-Equilibruim Thermodynamics. Field Theory and Varitional Principles, Springer-Verlag, BerlinHeidelberg-New York

[88] Cai,R.-G, and Kim,S.P, 2005, First law of thermodynamics and Friedmann equations of Friedmann-Robertson-Walker universe. JHEP, 02, 050-067

[89] Polyakov, A. M., 2010, Decay of Vacuum Energy. Nucl. Phys. B., 834, 316-329. 